\title{
Efeitos do óxido nitroso sobre parâmetros cardiovasculares e respiratórios em cães anestesiados com diferentes doses de desfluorano
}

\section{Effects of nitrous oxide on cardiovascular and respiratory parameteres in dogs anesthetized with different concentrations of desflurane}

\author{
Newton Nunes;* Celina Tie Nishimori; ${ }^{* *}$ Almir Pereira de Souza;*** Paulo Sérgio Patto dos Santos;**** \\ Danielli Parrilha de Paula;** Márlis Langenneger de Rezende;** Alessandra Valeiro Leite ${ }^{\star \star \star * *}$
}

\begin{abstract}
Resumo
Estudaram-se possíveis alterações cardiovasculares e respiratórias provocadas pelo desfluorano associado ou não ao $\mathrm{N}_{2} \mathrm{O}$. Para tal, utilizaram-se 30 cães adultos, machos ou fêmeas, sem raça definida e hígidos, distribuídos em dois grupos de igual número denominados GD e GDN. Os animais do GD receberam propofol para a indução anestésica e imediatamente após, $11,5 \mathrm{~V} \%$ de desfluorano diluído em $100 \%$ de $\mathrm{O}_{2}$. Decorridos 30 minutos do início da administração do anestésico volátil, o balão reservatório foi esvaziado, reduziu-se a concentração em 1,44V\% e o circuito anestésico foi saturado com a nova mistura. Repetiu-se o protocolo em intervalos de 15 minutos, até atingir a concentração anestésica equivalente a 8,64V\%. O GDN foi submetido ao mesmo protocolo, porém substituiu-se o fluxo diluente por $30 \% \mathrm{O}_{2}$ e $70 \% \mathrm{~N}_{2} \mathrm{O}$. Foram mensuradas as freqüências cardíaca ( $F C)$ e respiratória ( $F R$ ), pressões arteriais sistólica (PAS), diastólica (PAD) e média (PAM), concentração de dióxido de carbono ao final da expiração $\left(\mathrm{ETCO}_{2}\right)$ e saturação de oxi-hemoglobina $\left(\mathrm{SpO}_{2}\right)$. As mensurações foram realizadas antes da indução anestésica (M0), aos 30 (M30), 45 (M45) e 60 (M60) minutos de anestesia. Observou-se aumento da FC com discreta redução da PA e diminuição da FR com conseqüente aumento da $\mathrm{ETCO}_{2}$, coincidindo com a maior dose de desfluorano administrada. Concluiu-se que maiores concentrações de desfluorano induzem alterações discretas nas variáveis testadas e que a adição de $\mathrm{N}_{2} \mathrm{O}$ na mistura diluente não determina interferências significativas nos achados.
\end{abstract}

Palavras-chave: anestesia inalatória, desfluorano, óxido nitroso, cães.

\begin{abstract}
It was studied fortuitous cardiovascular and respiratory changes caused by desflurane associated or not to $\mathrm{N}_{2} \mathrm{O}$. Thirty healthy mongrel dogs, males or females, distributed in two equal groups denominated GD and GDN were used. The GD animals received propofol to anesthetic induction and after that, $11.5 \mathrm{~V} \%$ of desflurane diluted in $100 \% \mathrm{O}_{2}$. Thirty minutes after beginning the anesthetic volatile administration, the bag was emptied and concentration reduced to $1.44 \mathrm{~V} \%$, following saturation of anesthetic circuit with the new mixture. The protocol was repeated at each 15 minutes until obtain $8.64 \mathrm{~V} \%$ anesthetic concentration. The GDN animals were submitted to the same methodology, but the diluent flow was substituted by $30 \% \mathrm{O}_{2}$ and $70 \% \mathrm{~N}_{2} \mathrm{O}$. It was evaluated the heart and respiratory rates (HR and RR), systolic, diastolic and mean arterial pressures (SAP, DAP and MAP), end tidal carbon dioxide $\left(\mathrm{ETCO}_{2}\right)$ and oxyhemoglobin saturation $\left(\mathrm{SpO}_{2}\right)$. The parameters were measured before the induction of anesthesia (M0), 30 (M30), 45 (M45) and 60 (M60) after that. It was observed that HR increased with reduction on AP and RR decreased following $\mathrm{ETCO}_{2}$ increasing, coincident with the administration of desflurane in higher dose. It was concluded that higher concentration of desflurane induce little alterations on tested variations and the addition of $\mathrm{N}_{2} \mathrm{O}$ on the diluent mixture doesn't determine significant interferences on finding.
\end{abstract}

Keywords: inhalatory anesthesia, desflurane, nitrous oxide, dogs.

\footnotetext{
* Professor doutor - Departamento de Clínica e Cirurgia Veterinária, Faculdade de Ciências Agrárias e Veterinárias - UNESP - Campus de Jaboticabal - Via de Acesso: Prof. Paulo Donato Castellane s/n - Zona rural - Jaboticabal, SP - Brasil - CEP 14884-900 e-mail: newton@fcav.unesp.br - Tel.: (16) 3209-2626. (autor para correspondência).

** Pós-graduanda da Faculdade de Ciências Agrárias e Veterinárias - UNESP - Câmpus de Jaboticabal, SP.

*** Professor de Anestesiologia Veterinária - Universidade Federal do Tocantins - Campus de Araguaína, TO.

${ }^{* * * *}$ Professor de Anestesiologia Veterinária - Centro Universitário Barão de Mauá - Ribeirão Preto, SP.

***** Médica-veterinária, mestre em Cirurgia Veterinária.
} 


\section{Introdução}

Diversos fármacos têm sido analisados em associação aos agentes anestésicos inalatórios da atualidade, na tentativa de se obter uma anestesia balanceada de qualidade e segurança para os pacientes.

O desfluorano é um agente anestésico halogenado fluorinado, com peso molecular de 168 , ponto de ebulição de $23,5^{\circ} \mathrm{C}$, pressão de vapor de aproximadamente $664 \mathrm{mmHg}$ a $20^{\circ} \mathrm{C}$ e possui baixo coeficiente de solubilidade sangue/gás de 0,42 (Smiley, 1992), apresentando efeito anestésico somente em altas concentrações (Eger II, 1992), ao redor de 7,5V\% em cães e 6-7V\% no homem.

Este fármaco promove aumento da atividade simpática atingindo o pico máximo quando decorridos cinco minutos de exposição ao fármaco (Pacentine et al., 1995). Além disso, o desfluorano apresenta uma ação simpatomimética devido à existência de sítios receptores nas vias aéreas superiores, que respondem rapidamente à elevação da concentração deste gás nos alvéolos, devido ação irritante sobre a mucosa do trato respiratório (Muzi et al., 1996). Foi relatado que a utilização deste fármaco promove depressão da ventilação espontânea no homem, além de produzir decréscimo no volume minuto (VM) de maneira dependente da dose e aumento da freqüência respiratória (FR) (Lockhart et al., 1991).

No sistema cardiovascular, o desfluorano causa decréscimo da pressão arterial (PA), dependente da dose, resultante da diminuição da resistência vascular sistêmica (RVS) (Grundmann et al., 1996), promove aumento da freqüência cardíaca (FC) (Clarke et al., 1996a), além de deprimir a contratilidade (Pagel et al., 1991).

O óxido nitroso $\left(\mathrm{N}_{2} \mathrm{O}\right)$ é um gás incolor, não irritante, tem odor adocicado, não é inflamável e nem explosivo. Seu peso molecular é de aproximadamente 44, pode ser comprimido para o estado líquido abaixo de 50 atmosferas a $28^{\circ} \mathrm{C}$ e tem ponto de ebulição a $-89^{\circ} \mathrm{C}$. (Wylie e Churchill-Davidson, 1974). É um excelente analgésico, isento de propriedades hipnóticas, portanto, não induz planos anestésicos profundos. É potente o suficiente para promover uma analgesia correspondente a 10-15mg de morfina sem o risco de depressão respiratória (Stenqvist et al., 2001). Apresenta coeficiente de solubilidade sangue/gás de 0,47 e é rapidamente absorvido pelos alvéolos. É recomendado administrar pelo menos uma concentração de $30 \%$ de oxigênio ao utilizá-lo para fins anestésicos, a fim de se evitar hipoxia cerebral (Wylie e ChurchillDavidson, 1974).

$\mathrm{O} \mathrm{N}_{2} \mathrm{O}$ causa depressão, dependente da dose, da contratilidade diafragmática, devido a mudanças na distribuição e regulação do impulso nervoso nos músculos respiratórios (Warner et al., 1998). Portanto, seu uso deve ser evitado em pacientes com doenças respiratória ou neurológica preexistentes (Faroux et al., 2002).

Este gás anestésico tem efeito depressor direto e dependente da dose sobre o miocárdio, que pode ser compensado pela ativação simpática (Hohner e Reiz, 1994), a qual pode contribuir com aumento da incidência de arritmias cardíacas (Steffey, 1996).

Devido às qualidades desses dois agentes e a necessidade de se obter em associações que possibilitem minimizar o custo da anestesia e os possíveis efeitos colaterais objetivouse, com a realização deste estudo, avaliar os possíveis efeitos do óxido nitroso sobre parâmetros cardiovasculares e respiratórios em cães anestesiados com diferentes doses de desfluorano.

\section{Material e métodos}

Foram utilizados 30 cães adultos, machos e fêmeas, sem raça definida, evitando-se fêmeas prenhes ou em estro, considerados hígidos após exames clínico e laboratoriais, que foram distribuídos aleatoriamente em dois grupos de igual número $(n=15)$ previamente denominados GD e GDN. A média de peso dos animais foi de 9,45 $\pm 2,57 \mathrm{~kg}$ e 11,30 $\pm 2,84 \mathrm{~kg}$, no GD e GDN, respectivamente.

Nos animais do GD, induziu-se a anestesia administrandose propofol por via intravenosa (DIPRIVAN - ZENECA Farmacêutica do Brasil Ltda. - São Paulo, SP, Brasil), na dose de 8,9 $\pm 1,65 \mathrm{mg} / \mathrm{kg}$. Os cães foram intubados com sonda de Magill e imediatamente submetidos à anestesia inalatória pelo desfluorano (SUPRANE - ZENECA Farmacêutica do Brasil Ltda. - São Paulo, SP, Brasil), diluído em $100 \%$ de $\mathrm{O}_{2}$, em fluxo total de $30 \mathrm{~mL} / \mathrm{kg} / \mathrm{min}$, por meio de circuito anestésico tipo "semifechado" (OHMEDA - mod. Excel 210SE - DatexOhmeda - Miami, EUA), dotado de vaporizador (OHMEDA mod. TEC 6 - Datex-Ohmeda-Miami, EUA) termocompensado, microprocessado e calibrado para o agente anestésico.

Inicialmente, o desfluorano foi fornecido na concentração de 1,6 CAM (11,5V\%), considerando-se esta unidade como sendo equivalente a $7,2 \mathrm{~V} \%$ (Clarke et al., 1996a), mensurada em equipamento digital (OHMEDA - mod. RGM 5250 - DatexOhmeda - Miami, EUA). Após 30 minutos do início da administração do desfluorano, o balão reservatório do equipamento foi esvaziado e reduziu-se a concentração em 0,2 CAM $(1,44 \mathrm{~V} \%)$ e o circuito anestésico foi saturado novamente com a mistura gasosa. Aguardou-se a leitura da concentração expirada atingir aquela previamente determinada. Repetiu-se este protocolo, em intervalos de 15 minutos, até que fosse atingida a concentração anestésica equivalente a 1,2 CAM $(8,64 \mathrm{~V} \%)$. Utilizou-se colchão térmico ativo (Gaymar - mod. TP - 500 - Londres, Inglaterra) na tentativa de manter a temperatura corporal entre 38,3 e $39,0^{\circ} \mathrm{C}$.

Para os animais do GDN, adotou-se a mesma metodologia proposta para o GD, porém, utilizou-se como fluxo diluente $30 \%$ de $\mathrm{O}_{2}$ e $70 \%$ de $\mathrm{N}_{2} \mathrm{O}$.

Foram avaliados os valores de freqüência cardíaca, obtida em monitor de eletrocardiografia computadorizado (TEB mod. ECGPC software versão 1.10 - São Paulo, SP, Brasil); pressões arteriais sistólica, diastólica e média (PAS, PAD e PAM, respectivamente), por meio de monitor multiparamétrico não-invasivo (Digimax 5000 - mod. ESFMN 2T - São Paulo, $\mathrm{SP}$, Brasil) em M0, e a partir de M30, pelo método invasivo, após cateterização da artéria femoral esquerda ou direita, cuja leitura foi feita em monitor digital computadorizado (DIXTAL mod. DX 2010 - Manaus, AM, Brasil); freqüência respiratória (FR) e concentração de dióxido de carbono ao final da expiração $\left(\mathrm{ETCO}_{2}\right)$, obtidas por leitura direta em monitor multiparamétrico (OHMEDA - mod. RGM 5250 - Datex-Ohmeda - Miami, EUA), adaptando-se o sensor de aspiração à máscara facial em M0 e conectando-o entre a sonda orotraqueal e o equipamento 
de anestesia, nos demais momentos; saturação da oxihemoglobina $\left(\mathrm{SpO}_{2}\right)$ obtida por leitura direta em monitor multiparamétrico, sendo o emissor/sensor adaptado em região cutânea compatível com a sensibilidade do dispositivo em M0 e na língua a partir de M30.

As mensurações foram realizadas imediatamente antes da indução da anestesia (M0), decorridos 30 minutos do estabelecimento da concentração inicial do agente volátil (M30) e as demais coincidiram com o estabelecimento das concentrações de 1,4 e 1,2 CAM, respectivamente M45 e M60.

A avaliação estatística das variáveis foi efetuada por meio de Análise de Perfil (Morrison, 1967; Curi, 1980), considerando um nível de significância de 5\% $(p<0,05)$.

\section{Resultados e discussão}

Houve elevação da FC em ambos os grupos a partir de M30, sendo que em GD as médias permaneceram no mesmo patamar após esse momento, enquanto no GDN as médias continuaram a ascender até o término do período experimental. O aumento da FC concomitante à administração de desfluorano corrobora os achados de Zwass et al. (1992), Pacentine et al. (1995), Clarke et al. (1996b) e Park (2002), que relataram ser este efeito decorrente da atividade simpatomimética do desfluorano. Estes achados vão ao encontro dos de Clarke et al. (1996a), que afirmaram que a variação da FC durante a anestesia pelo desfluorano não é dependente da dose. Devido aos conhecidos efeitos simpatomiméticos do óxido nitroso (Hohner e Reiz, 1994; Mutoh et al., 2001), poder-se-ia esperar um efeito aditivo no incremento da FC em GDN, quando comparado à administração isolada do desfluorano. Contudo, este fenômeno não foi observado, uma vez que os valores deste parâmetro foram similares nos dois grupos. No GDN houve aumento progressivo da FC em M45 e M60, quando comparados ao momento inicial da administração dos fármacos. Este fenômeno coincidiu com a redução da concentração de desfluorano, sugerindo que a alta concentração desse agente anestésico poderia estar inibindo a ação simpatomimética do $\mathrm{N}_{2} \mathrm{O}$ e que com a administração de doses menores permitiu observar o suposto efeito aditivo de ambos os agentes, resultando no incremento desses valores ao longo do período experimental.

A comparação da PAD entre grupos mostrou que em M0, os animais do GD apresentaram médias maiores que os do GDN. O fato deste fenômeno ter ocorrido no momento basal, antes da administração de qualquer agente, descarta qualquer influência dos fármacos em teste, sendo este resultado decorrente dos animais do GDN apresentarem pressões arteriais diastólicas iniciais menores que os do GD. Na análise individual dos grupos, observou-se que no GD, houve redução das pressões arteriais em M30, embora não significativa, coincidindo com a administração da maior concentração do desfluorano (Grundmann et al., 1996; Park, 2002). No GDN, apesar da redução dos valores das pressões arteriais em M30, esta foi significativa apenas para a PAS. A partir deste momento, todas as pressões comportaram-se de maneira similar, tendendo a retornar progressivamente aos valores iniciais ao longo dos momentos. Essas discretas alterações podem ser explicadas devido à administração de altas con- centrações de desfluorano, em associação ao óxido nitroso, diminuir a pressão arterial em razão da depressão na contratilidade miocárdica ocasionada pelos agentes inalatórios, como descrito por Cahalan et al. (1991) e da redução na RVS (Grundmann et al., 1996).

Com relação à $\mathrm{FR}$, ficou evidente que a ação do desfluorano sobre este parâmetro respiratório é dependente da dose, uma vez que a média de M30 em ambos os grupos, correspondente à maior concentração de desfluorano, apresentou-se menor que as demais. A partir desse momento, as médias comportaram-se de maneira ascendente, na medida em que as concentrações do agente volátil diminuíram, como citado por Patel e Goa (1995) e Steffey (1996). Embora o óxido nitroso deprima os centros respiratórios (Amaral, 2001) e cause depressão da contratilidade diafragmática (Fauroux et al., 2002), ambos os efeitos dependentes da dose, neste estudo verificou-se que apesar de terem sido mantidas altas concentrações de óxido nitroso de forma constante durante todo o experimento, a FR aumentou gradativamente a partir de M30. Isso sugere que a diminuição deste parâmetro foi causada principalmente pela alta concentração de desfluorano empregada, indicando que este agente inalatório é um depressor respiratório mais potente do que o óxido nitroso.

A ETCO ${ }_{2}$, que auxilia na monitoração da ventilação, comportou-se de maneira inversamente proporcional à $F R$, como esperado, visto que as mesmas apresentam estreita correlação. No entanto, apesar da recuperação dos valores de FR, os valores de $\mathrm{ETCO}_{2}$ no GDN foram maiores que os do GD em M60. Este fenômeno pode ser explicado pela eventual depressão da contratilidade diafragmática causada pelo óxido nitroso (Fauroux et al., 2002) que, conseqüentemente, ocasionaria diminuição da amplitude respiratória. Em ambos os grupos houve aumento da média de $\mathrm{ETCO}_{2}$ em $\mathrm{M} 30$, coincidindo com o momento da administração da concentração mais elevada de desfluorano e com a redução da $F R$, de acordo com investigações prévias realizadas por Steffey (1996) e Rezende (2000). Após M30, esses valores foram retornando aos valores iniciais, concomitantemente à redução das concentrações anestésicas, devido ao menor efeito depressor no sistema respiratório decorrente da administração de doses menores do anestésico (Clarke et al., 1996a; Nunes et al., 2001).

A oximetria reflete a percentagem de oxi-hemoglobina saturada por oxigênio, determinando alto grau de previsibilidade da hipoxia tissular, o que permite redução no índice de acidentes anestésicos relacionados com a hipoxemia (Nunes, 2002). Sendo assim, as diferenças encontradas entre os grupos em M30 e M45, cujo GD apresentou valores médios maiores que o GDN, são de valor clínico questionável, pois além das médias serem similares, os valores mantiveram-se dentro da faixa de normalidade para a espécie (Moyle et al., 1994). A avaliação individual dos grupos mostrou aumento das médias, quando comparados aos valores basais. Entretanto, podem ser aceitas as mesmas considerações realizadas para a interpretação dos resultados entre grupos, além do quê, neste caso, as variações encontradas poderiam estar relacionadas à maior concentração de oxigênio fornecida ao animal durante o período anestésico, quando comparada à concentração de oxigênio do ar ambiente. 
Tabela 1 - Valores médios e desvios-padrão $(x \pm s)$ das variáveis: freqüência cardíaca (FC), pressões arteriais sistólica, diastólica e média (PAS, PAD e PAM, respectivamente), freqüência respiratória (FR), concentração de dióxido de carbono ao final da expiração $\left(\mathrm{ETCO}_{2}\right)$ e saturação da oxi-hemoglobina $\left(\mathrm{SpO}_{2}\right)$, obtidos em cães anestesiados com doses decrescentes de desfluorano, associado (GDN) ou não (GD) ao óxido nitroso, por um período de 60 minutos

\begin{tabular}{|c|c|c|c|c|c|}
\hline VARIÁVEIS & & MO & M30 & M45 & M60 \\
\hline \multirow{2}{*}{ FC (batimentos/min.) } & GD & $111 \pm 18,96^{\mathrm{a}}$ & $132 \pm 22,61^{b}$ & $133 \pm 21,49^{b}$ & $134 \pm 22,48^{b}$ \\
\hline & GDN & $115 \pm 19,86^{\mathrm{a}}$ & $133 \pm 19,66^{b}$ & $139 \pm 21,63^{c}$ & $143 \pm 23,71^{\mathrm{C}}$ \\
\hline \multirow{2}{*}{$\begin{array}{c}\text { PAS } \\
(\mathrm{mmHg})\end{array}$} & GD & $131 \pm 17,19^{a}$ & $122 \pm 30,12^{a b}$ & $130 \pm 28,91^{a b}$ & $138 \pm 30,09^{\mathrm{ac}}$ \\
\hline & GDN & $125 \pm 10,79^{a}$ & $110 \pm 19,66^{b}$ & $125 \pm 18,92^{\mathrm{a}}$ & $139 \pm 19,51^{\mathrm{a}}$ \\
\hline \multirow{2}{*}{$\begin{array}{c}\text { PAD } \\
(\mathrm{mmHg})\end{array}$} & GD & $79 \pm 14,12^{* a}$ & $69 \pm 24,48^{\mathrm{ab}}$ & $75 \pm 22,49^{\mathrm{ac}}$ & $82 \pm 18,85^{\mathrm{ad}}$ \\
\hline & GDN & $69 \pm 8,87^{\mathrm{a}}$ & $61 \pm 14,75^{a b}$ & $75 \pm 13,79^{\text {ac }}$ & $83 \pm 11,00^{d}$ \\
\hline \multirow{2}{*}{$\begin{array}{c}\text { PAM } \\
(\mathrm{mmHg})\end{array}$} & GD & $99 \pm 15,42^{a}$ & $89 \pm 26,54^{a b}$ & $97 \pm 24,64^{\mathrm{ac}}$ & $105 \pm 23,53^{\text {ad }}$ \\
\hline & GDN & $91 \pm 9,74^{a}$ & $82 \pm 16,37^{\mathrm{ab}}$ & $96 \pm 14,88^{\mathrm{ac}}$ & $107 \pm 13,74^{d}$ \\
\hline \multirow{2}{*}{ FR (movimentos/min.) } & GD & $28 \pm 9,70^{a}$ & $15 \pm 8,03^{b}$ & $21 \pm 18,11^{\mathrm{c}}$ & $26 \pm 20,85^{\mathrm{ac}}$ \\
\hline & GDN & $23 \pm 6,35^{a}$ & $11 \pm 6,05^{b}$ & $16 \pm 7,60^{c}$ & $19 \pm 8,66^{\mathrm{ac}}$ \\
\hline \multirow{2}{*}{$\begin{array}{c}\mathbf{E T C O}_{2} \\
(\mathrm{mmHg})\end{array}$} & GD & $28,33 \pm 5,80^{a}$ & $44,07 \pm 13,05^{b}$ & $37,40 \pm 9,59^{c}$ & $33,07 \pm 8,34^{*} \mathrm{c}$ \\
\hline & GDN & $30,80 \pm 6,26^{a}$ & $45,60 \pm 17,71^{b}$ & $41,47 \pm 15,88^{\mathrm{C}}$ & $42,07 \pm 9,40^{b c}$ \\
\hline \multirow{2}{*}{$\mathrm{SpO}_{2}(\%)$} & GD & $97,20 \pm 1,66^{a}$ & $99,87 \pm 0,35^{* b}$ & $100 \pm 0^{* 6}$ & $99,53 \pm 1,55^{b}$ \\
\hline & GDN & $97,67 \pm 1,72^{a}$ & $99,20 \pm 0,77^{b}$ & $99,33 \pm 1,17^{b}$ & $99,33 \pm 1,17^{b}$ \\
\hline
\end{tabular}

* Existe diferença estatística entre os grupos para este momento pela Análise de Perfil $(p<0,05)$.

Médias seguidas por letras diferentes, nas linhas, diferem significativamente entre si pela Análise de Perfil $(p<0,05)$.

\section{Conclusões}

Foi possível concluir que maiores concentrações de desfluorano induzem alterações discretas nas variáveis testadas e que a adição de $\mathrm{N}_{2} \mathrm{O}$ na mistura diluente não determina interferências significativas nos achados.

\section{Agradecimentos}

Os autores agradecem a Fundação de Amparo à Pesquisa do Estado de São Paulo (FAPESP), pelo apoio financeiro concedido.

\section{Referências}

AMARAL, J. L. G. Anestesia inalatória. In: YAMASHITA, A. M.; TAKAOKA, F.; AULER JUNIOR, J. O. C.; IWATA, N. M. (Ed.). Anestesiologia-SAESP. 5. ed. São Paulo: Atheneu, 2001, p. 551-577. CAHALAN, M. K.; WEISKOPF, R. B.; EGER II, E. I.; YASUDA, N.; IONESCU, P.; RAMPIL, I. J.; LOCKHART, S. H.; FREIRE, B.; PETERSON, N. A. Hemodynamic effects of desflurane/nitrous oxide anesthesia in volunteers. Anesth. Analg., v. 73, n. 2, p. 157-164, 1991.

CLARKE, K. W.; ALIBHAI, H. I. K.; LEE, Y. H.; HAMMOND, R. A. Cardiopulmonary effects of desflurane in the dog during spontaneous and artificial ventilation. Res. Vet. Sci., v. 61, n. 1, p. 82-86, $1996 a$.

CLARKE, K. W.; SONG, D. Y.; ALIBHAI, H. I. K.; LEE, Y. H. Cardiopulmonary effects of desflurane in ponies, after induction of anaesthesia with xylazine and ketamine. Vet. Rec., v. 139, p. 180-185, 1996b.

CURI, P. R. Análise de medidas repetidas em experimentos biológicos. Rev. Bras. Estat., v. 41, n. 161, p. 137-150, 1980.

EGER II, E. I. Desflurane animal and human pharmacology: aspects of kinetics, safety, and MAC. Anesth. Analg., v. 75, suppl., p. 3-9, 1992. FAUROUX, B.; CORDINGLEY, J.; HART, N.; CLÉMENT, A.; MOXHAM, J.; LOFASO, F.; POLKEY, M. I. Depression of diaphragm contractility by nitrous oxide in humans. Anesth. Analg., v. 94, n. 2, p. 340-345, 2002. GRUNDMANN, U.; MULLER, M.; KLEINSCHMIDT, S.; LARSEN, B.; LARSEN, R. Cardiovascular effects of desflurane and isoflurane in patients with coronary artery disease. Acta Anaesthesiol. Scand., v. 40 , n. 9 , p. $1101-1107,1996$.
HOHNER, P.; REIZ, S. Nitrous oxide in the cardiovascular system. Acta Anaesthesiol. Scand., v. 38, n. 8, p. 763-766, 1994.

LOCKHART, S. H.; RAMPIL, I. J.; YASUDA, N.; EGER II, E. I.;WEISKOPF, R. B. Depression of ventilation by desflurane in humans. Anesthesiology, v. 74, n. 3, p. 484-488, 1991.

MORRISON, D. F. Multivariate statistical methods. New York: Mac Graws Hill Book, 1967.

MOYLE, J. T. B.; HAHN, C. E. W.; ADAMS, A. P. Pulse oximetry principles and practice series. London: BMJ, 1994.

MUTOH, T.; NISHIMURA, R.; SASAKI, N. Effects of nitrous oxide on mask induction of anesthesia with sevoflurane or isoflurane in dogs. Am. J. Vet. Res., v. 62, n. 11, p. 1727-1733, 2001.

MUZI, M.; EBERT, T. J.; HOPE, W. G.; ROBINSON, B. J.; BELL, L. B. Site(s) mediating sympathetic activation with desflurane. Anesthesiology, v. 85, n. 4, p. 737-747, 1996.

NUNES, N. Monitoração da anestesia. In: FANTONI, D. T.; CORTOPASSI, S. R. G. (Ed.). Anestesia em cães e gatos. São Paulo: Roca, 2002, p. 64-81.

NUNES, N.; SANTOS, P. S. P.; VICENTI, F. A. M.; REZENDE, M. L.; MARTINS, S. E. C. Efeitos da associação de fentanil e droperidol na concentração alveolar mínima do desfluorano, em cães. Ars Vet., v. 17, n. 2, p. 86-92, 2001.

PACENTINE, G. G.; MUZI, M.; EBERT, T. J. Effects of fentanyl on sympathetic activation associated with the administration of desflurane. Anesthesiology, v. 82, n. 4, p. 823-831, 1995. 
PAGEL, P. S.; KAMPINE, J. P.; SCHMELING, W. T.; WALTIER, D. C. Comparison of the systemic and coronary hemodynamic actions of desflurane, isoflurane, halothane, and enflurane in the chronically instrumented dog. Anesthesiology, v. 74, n. 3, p. 539-551, 1991.

PARK, K. W. Cardiovascular effects of inhalation anesthetics. Inter. Anesth. Clin., v. 40, n. 1, p. 1-14, 2002.

PATEL, S. S.; GOA, K. L. Desflurane: a review of its pharmacodynamic and pharmacokinetic properties and its efficacy in general anaesthesia. Drugs, v. 50, n. 4, p. 742-767, 1995.

REZENDE, M. L. Dinâmica cardiorrespiratória e concentração alveolar mínima do desfluorano, em cães pré-tratados com levomepromazina. 2000. 90 f. Dissertação (Mestrado) - Faculdade de Ciências Agrárias e Veterinárias - Universidade Estadual Paulista, 2000.

SMILEY, R. M. An overview of induction and emergence characteristics of desflurane in pediatric, adult, and geriatric patients. Anesth. Analg., v. 75, suppl. 4, p. 38-46, 1992.
STEFFEY, E. P. Inhalation anesthetics. In: THURMON, J. C.; TRANQUILLI, W. J.; BENSON, G. J. (Ed.). Lumb \& Jones' veterinary anesthesia. 3. ed. Philadelphia: Lea \& Febiger, 1996, p. 297-329.

STENQVIST, O.; HUSUM, B.; DALE, O. Nitrous oxide: an ageing gentleman. Acta Anaesthesiol. Scand., v. 45, n. 2, p. 135-137, 2001.

WARNER, D. O.; WARNER, M. A.; JOYNER, M. J.; RITMAN, E. L. The effect of nitrous oxide on chest wall function in humans and dogs. Anesth. Analg., v. 86, n. 5, p. 1058-1064, 1998.

WYLIE, W. D.; CHURCHILL-DAVIDSON, H. C. Os gases anestésicos. In: WYLIE, W. D.; CHURCHILL-DAVIDSON, H. C. (Ed.). Anestesiologia. 3. ed. Rio de Janeiro: Guanabara Koogan, 1974, p. 184-195.

ZWASS, M. S.; FISHER, D. M.; WELBORN, L. G.; COTÉ, C. J.; DAVIS, P.J.; DINNER, M.; HANNALLAH, R.S.; LIU, L. M. P.; SARNER, J.; McGILL, W. A.; ALIFIMOFF, J. K.; EMBREEE, P. B.; RYAN COOK, D. Induction and maintenance characteristics of anesthesia with desflurane and nitrous oxide in infants and children. Anesthesiology, v. 76, n. 3, p. 373-378, 1992. 